\title{
Analysis of the Hexagonal Split Ring Resonator using Machine Learning by Considering Split Gap as a Prime Factor
}

\author{
${ }^{1}$ Thippesha D and ${ }^{2}$ Pramodh BR \\ ${ }^{1}$ ECE department Govt. Engineering college, \\ Huvinahadagali, India; \\ ${ }^{2}$ Data Scientist, Bangalore, India \\ Email: \{' thippesh790@gmail.com, \\ pramodhbr29@gmail.com \}
}

\begin{abstract}
The Hexagonal split-ring resonators (HSRR) are one of the prime elements of metamaterial and patch antenna design in the millimetre-wave range. Even though it's widely used there is no particular mathematic model is available for it. This analysis presents the mathematical nature of the relation between split widths, resonance frequencies; reflection (s11) and mutual coupling (s12) by identifying tend of the data with the aid of machine learning algorithms. The predicted relation will help to design efficient metamaterial, antennas and related appliances.
\end{abstract}

\section{Key Words}

Hexagonal split-ring resonators, Metamaterial, Patch antenna, Mathematical Analysis, Machine Learning, Resonance Frequencies, Linear Regression, Decision tree, Random Forest, Polynomial Regression.

\section{Introduction}

The metamaterials are the revolutionary materials that boosted the electromagnetic dives ability to a new level, from wireless communication[1-4] to radio astronomy they play a very important role. One such material is hexagonal split Ring resonators, it is used in radar, radio astronomy, and antennas and sensors. Even after being employed in multiple applications, there is no particular mathematical model.

There exist a generalized Mathematical Modeling for Nsided Regular Polygon Split Ring Resonator(NRPSRR)[5] but it doesn't explain the frequency hopping in a rotating HSRR. This work concentrate on all the aspects which are affected by the split gap in particular to HSRR. The analysis also tries to address the frequency hopping in the rotating HSRR and methods to effectively design the HSRR for the required frequency.

Machine learning is a combination of statistics and computer programing which was created for analyzing complex data. It simplifies the statistical operations and coding needed. There are many algorithms are there in machine learning, this analysis uses only four of them, the algorithms were selected based on flexibility and accuracy[6-9].

\section{Methodology}

\subsection{Experiment Setup}

an FR4 substrate is placed between two ports as shown in Fig. 1(a)(b). In this setup only one port will act as a Tx port another will be an Rx port. At first, the split gap of the ring is varied and respective data is recorded, in the second stage the split will be constant but the ring will be tilted with a step angle of 300 the experiment will continue up to 3600 for each step the resonance frequency is noted down. This experiment is carried out because the change in current density is observed when the angle of the ring changes as shown in Fig. 2. The experiment was carried out with three different sizes of rings with similar ring widths. Table 1 . shows different parameters of rings used, the frequency range of $1 \mathrm{GHz}$ to $7 \mathrm{GHz}$ is used to test the ring.

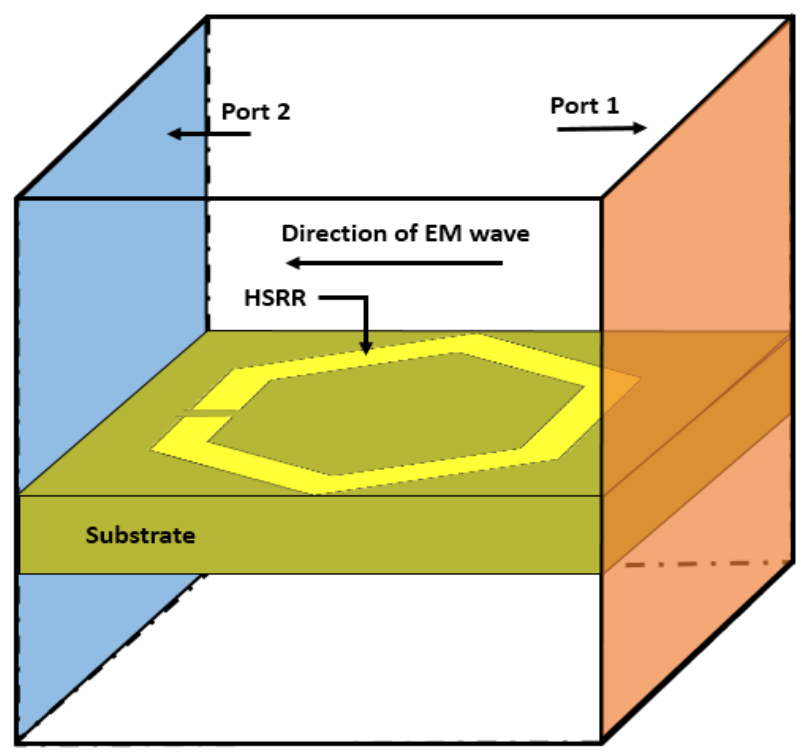

(a) 


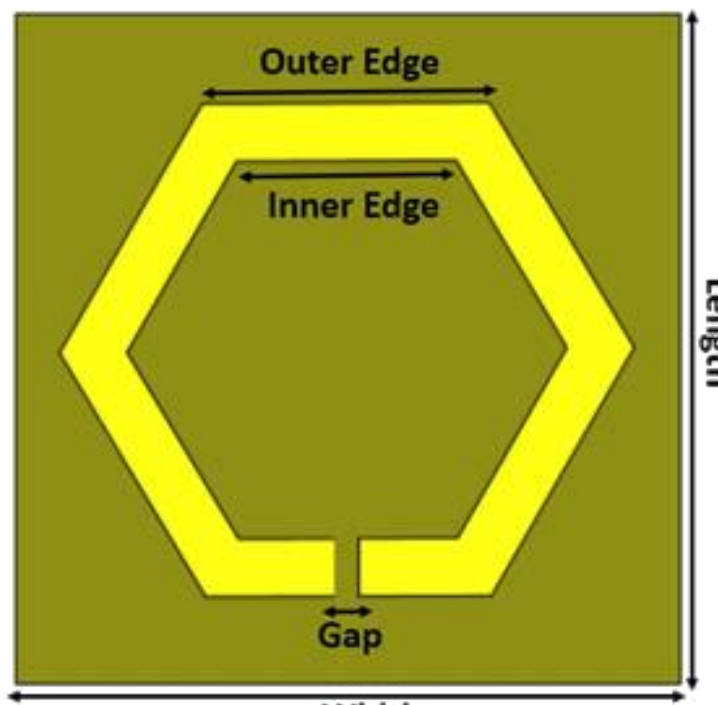

Width

(b)

Fig. 1. Experiment setup (a)arrangement of the ring, (b)HSRR design parameters.

Table 1. The Parameter Variations

\begin{tabular}{cccc}
\hline \multicolumn{4}{c}{ Change in Split Gap } \\
\hline & $\begin{array}{c}\text { Outer Edge } \\
\text { in mm }\end{array}$ & $\begin{array}{c}\text { Inner Edge } \\
\text { in mm }\end{array}$ & $\begin{array}{c}\text { Split Gap } \\
\text { in mm }\end{array}$ \\
\hline HSRR 1 & 4 & 3 & $0.01-0.3$ \\
HSRR 2 & 4.25 & 3.25 & $0.01-0.3$ \\
HSRR 3 & 3.25 & 2.25 & $0.01-0.3$ \\
& & & \\
\hline
\end{tabular}

Change in Angle of HSRR

\begin{tabular}{cccc}
\hline & $\begin{array}{c}\text { Outer Edge } \\
\text { in mm }\end{array}$ & $\begin{array}{c}\text { Inner Edge } \\
\text { in mm }\end{array}$ & $\begin{array}{c}\text { Split Gap in } \\
\text { mm }\end{array}$ \\
\hline HSRR 1 & 4 & 3 & 0.3 \\
HSRR 2 & 4.25 & 3.25 & 0.3 \\
HSRR 3 & 3.25 & 2.25 & 0.3 \\
\hline
\end{tabular}

\subsection{Data analysis using the machine learning algorithms}

A set of Four Regression algorithms from machine learning are considered for the analysis, Regression is a method of predicting using statistical observations, the selected algorithms are Linear Regression, Decision tree learning, random forest learning and polynomial regression is used.

Linear Regression is a linear approach to describe the relationship between the independent variable and dependent variable, the relationship between this variable will be presented in the form of a linear equation.

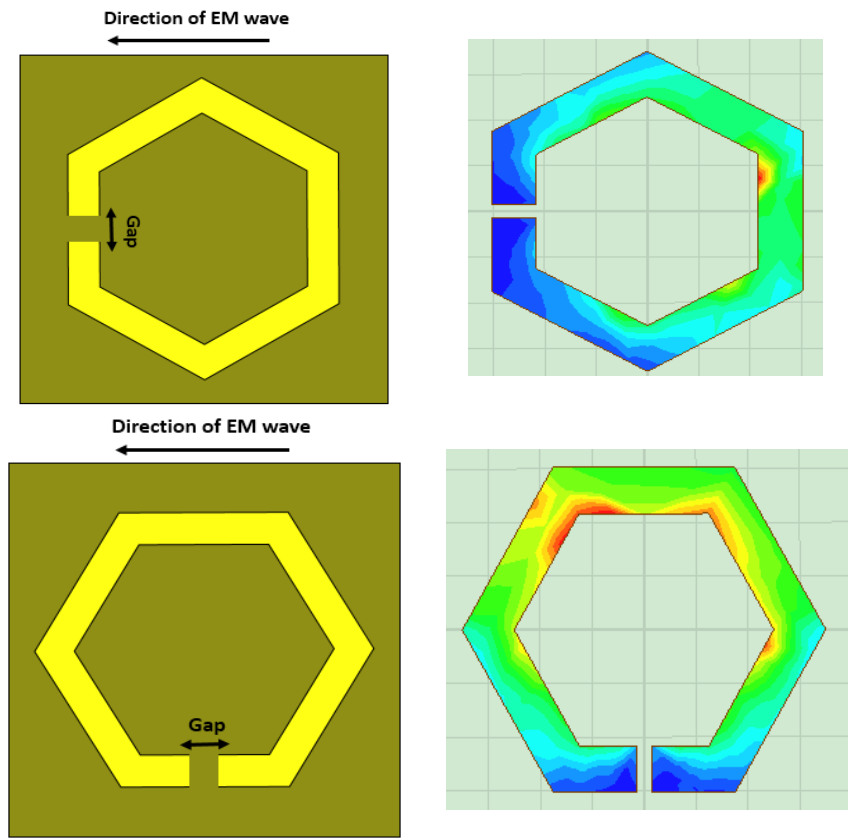

Fig. 2. The effect of rotation of HSRR in the EM field.

The linear regression equation is

$$
y=X \beta+\epsilon
$$

Where y dependent matrix, $\mathrm{x}$ is the independent matrix, is a matrix of coefficients and is a matrix of the error term.

A decision tree is a predictive approach to describe the target as a set of observations. These observations may have numerical values or Boolean values. It works for regression as well as classification Fig. 3.

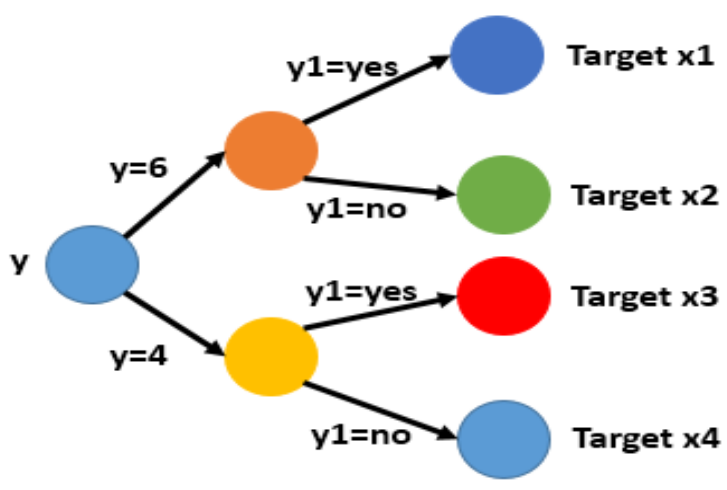

Fig. 3. Decision Tree algorithm flow

Random Forest is a method that uses multiple decision trees of different classes for a given classification or a regression operation. It works for regression as well as classification Fig. 4. 


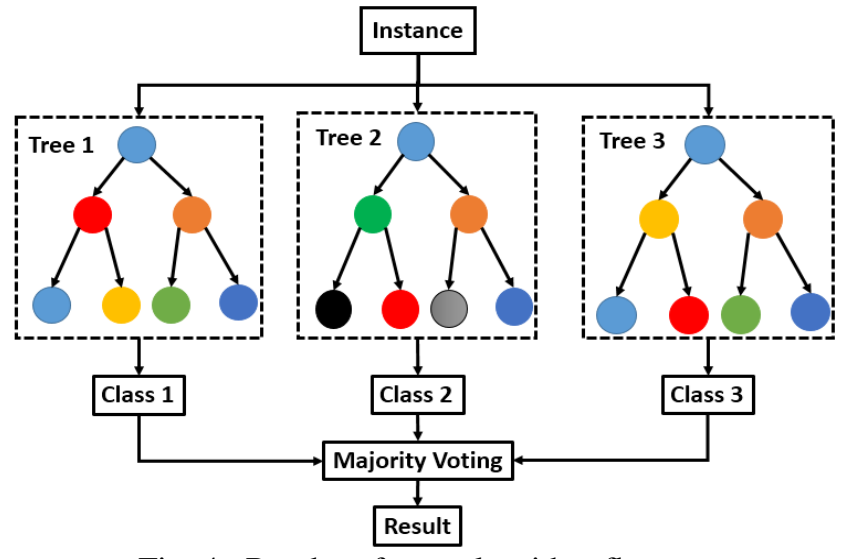

Fig. 4. Random forest algorithm flow

Polynomial Regression is a polynomial approach to describe the relationship between an independent variable and dependent variable, the relationship between these variables will be presented in the form of a polynomial equation with a degree of the polynomial.

The general form of the polynomial regression equation is

$$
\begin{gathered}
y=\beta_{0}+\beta_{1} x+\beta_{2} x^{2}+\beta_{3} x^{3}+\cdots+\beta_{n} x^{n}+\varepsilon \\
\text { For } i=1,2 \ldots n .
\end{gathered}
$$

Where $\mathrm{x}$ is an independent variable, $\mathrm{y}$ is a dependent variable and $\beta$ is a coefficient and $\varepsilon$ is an error term.

\section{RESULTS}

The obtained data is analyzed with aid of a machine learning algorithm and the results are as follows.

Table 2. Accuracy of the Prediction

\begin{tabular}{lccc}
\hline & $\begin{array}{c}\text { Linear } \\
\text { Regression }\end{array}$ & Decision tree & $\begin{array}{c}\text { Random } \\
\text { Forest }\end{array}$ \\
\hline HSRR 1 & 92.41 & 86.51 & 89.06 \\
HSRR 2 & 85.65 & 88.9 & 92.91 \\
HSRR 3 & 82.29 & 80.65 & 90.3 \\
\hline
\end{tabular}

Table 3. RMSE of the Prediction

\begin{tabular}{cccc}
\hline & $\begin{array}{c}\text { Linear } \\
\text { Regression }\end{array}$ & $\begin{array}{c}\text { Decision } \\
\text { tree }\end{array}$ & $\begin{array}{c}\text { Random } \\
\text { Forest }\end{array}$ \\
\hline HSRR 1 & 0.00052 & 0.001266 & 0.00102 \\
HSRR 2 & 0.000883 & 0.000966 & 0.00066 \\
HSRR 3 & 0.00166 & 0.00134 & 0.00038 \\
\hline
\end{tabular}

\subsection{Change in Split Gap}

In this phase, the predictions were made using linear regression, decision tree and random forest algorithms. The accuracy results were tabulated in Table 2. And the Root Mean Square Error (RMSE) results are tabulated in Table 3. The random forest gives the best accuracy and with a very low error percentage compared to other algorithms. The linear regression can be considered as the second-best because even though it has accuracy but the error percentage is also high compared to random forest. The accuracy and results were plotted for comparison Fig. 5.

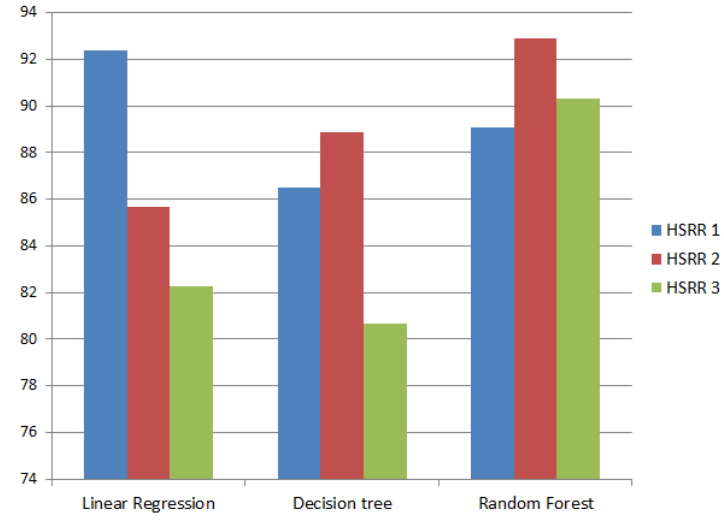

(a)

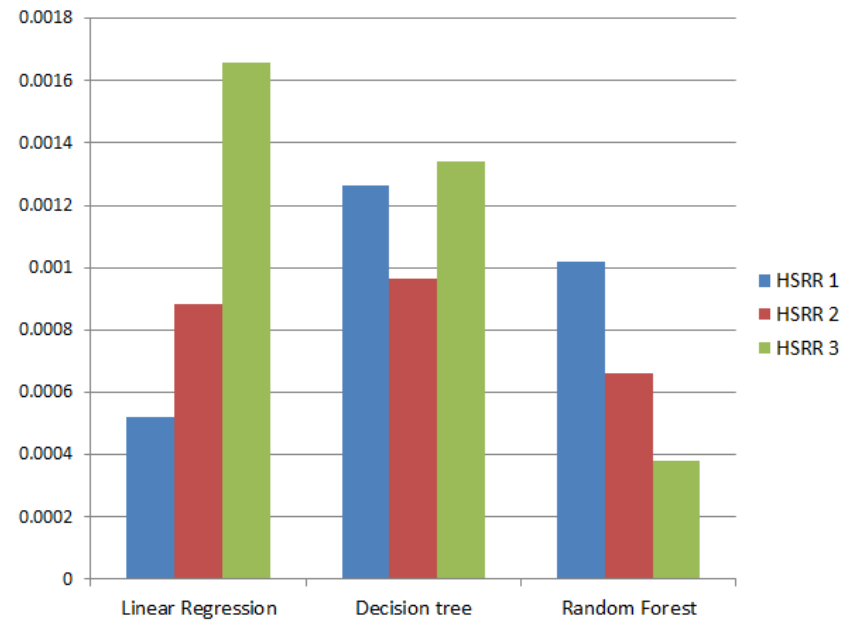

(b)

Fig. 5. Results plot (a) Accuracy, (b) Root mean square error.

\subsection{Change angle of the HSRR}

The rotation data appears to be polynomial hence the polynomial regression is used to analyze the data. The bestfitting line for the HSRR 1 data is as shown in Fig. 6(a)the equation of the line is (3) with an accuracy of $96.33 \%$. $y=4 E-05 x^{6}-0.0019 x^{5}+0.0294 x^{4}-0.2054 x^{3}+0.5915 x^{2}-$

$$
0.3418 \mathrm{x}+3.6308
$$

The best-fitting line for the HSRR 2 data is as shown in Fig. 6(b) the equation of the line is (4) with an accuracy of $90.59 \%$.

$$
\begin{array}{r}
y=1 E-04 x^{6}-0.004 x^{5}+0.062 x^{4}-0.4553 x^{3}+1.5633 x^{2}- \\
2.1321 x+4.4888
\end{array}
$$

The best-fitting line for the HSRR 3 data is as shown in Fig. 6(c) the equation of the line is (5) with an accuracy of $83.25 \%$.

$$
\begin{array}{r}
y=0.0001 x^{6}-0.0056 x^{5}+0.0946 x^{4}-0.7731 x^{3}+3.1377 x^{2}- \\
5.6734 x+8.0846
\end{array}
$$

\section{Conclusion}

Machine learning can be used to analyze the complex phenomenon of electromagnetics, these phenomena often produce results hard to summarize by simple mathematic models. These prediction models help to develop efficient antennas and radar applications using HSRR. 


\section{References}

[1] Visser, H. J. (2012). Antenna theory and applications. John Wiley \& Sons.

[2] M. A. ul Haq, S. Koziel and Q. S. Cheng, "A Novel Isolation Improvement Technique for Wideband MIMO Antenna Systems," 2019 13th European Conference on Antennas and Propagation (EuCAP), Krakow, Poland, 2019, pp. 1-4.

[3] T. Cui, X. Zhu, J. Zhang and Z. Zheng, "Circular Antenna Array Synthesis Technique Including Mutual Coupling Using Unitexcitation Active Element Pattern," 2018 12th International Symposium on Antennas, Propagation and EM Theory (ISAPE), Hangzhou, China, 2018, pp. 1-3,

[4] W. Zhang and Z. S. He, "Comments on "Waveform Optimization for Transmit Beamforming With MIMO Radar Antenna Array"," in IEEE Transactions on Antennas and Propagation, vol. 66, no. 11, pp. 6463-6463, Nov. 2018

[5] Y. Liu, Y. Jiao, Z. Weng, C. Zhang and H. Zhang, "A high-gain dielectric resonator antenna array fed by back-cavity," 2018 International Workshop on Antenna Technology (iWAT), Nanjing, 2018, pp. 1-3.

[6] N. Amral, C. S. Ozveren and D. King, "Short term load forecasting using Multiple Linear Regression," 2007 42nd International Universities Power Engineering Conference, Brighton, 2007, pp. 1192-1198, doi: 10.1109/UPEC.2007.4469121.

[7] H. Shakouri, G. R. Nadimi and F. Ghaderi, "Fuzzy linear regression models with absolute errors and optimum uncertainty," 2007 IEEE International Conference on Industrial Engineering and Engineering Management, Singapore, 2007, pp. 917-921, doi: 10.1109/IEEM.2007.4419325.

[8] X. Chen, "Recursive local polynomial regression estimation and its applications," Proceedings of the 31st Chinese Control Conference, Hefei, 2012, pp. 2043-2048.

[9] A. V. Omelchenko and O. V. Fedorov, "Polynomial regression coefficients estimation in finite differences space," 2015 25th International Conference Radioelektronika (RADIOELEKTRONIKA), Pardubice, 2015, pp. 257-260, doi: 10.1109/RADIOELEK.2015.7129024. 